\title{
Bryophyte colonization history of the virgin volcanic island Surtsey, Iceland
}

\author{
G. V. Ingimundardóttir ${ }^{1,2}$, H. Weibull ${ }^{3}$, and N. Cronberg ${ }^{1}$ \\ ${ }^{1}$ Department of Biology, Lund University, Ecology building, 22362 Lund, Sweden \\ ${ }^{2}$ Icelandic Institute of Natural History, Urriðaholtsstræti 6-8, 212 Garðabær, Iceland \\ ${ }^{3}$ Naturcentrum AB, Strandtorget 3, 44430 Stenungsund, Sweden \\ Correspondence to: N. Cronberg (nils.cronberg@biol.lu.se)
}

Received: 9 January 2014 - Published in Biogeosciences Discuss.: 26 March 2014

Revised: 18 June 2014 - Accepted: 4 July 2014 - Published: 20 August 2014

\begin{abstract}
The island Surtsey was formed in a volcanic eruption south of Iceland in 1963-1967 and has since then been protected and monitored by scientists. The first two moss species were found on Surtsey as early as 1967 and several new bryophyte species were discovered every year until 1973 when regular sampling ended. Systematic bryophyte inventories in a grid of $100 \mathrm{~m} \times 100 \mathrm{~m}$ quadrats were made in 1971 and 1972: the number of observed species doubled, with 36 species found in 1971 and 72 species in 1972. Here we report results from an inventory in 2008, when every other of the grid's quadrats were searched for bryophytes. Despite lower sampling intensity than in 1972, distributional expansion and contraction of earlier colonists was revealed as well as the presence of new colonists. A total of 38 species were discovered, 15 of those were not encountered in 1972 and eight had never been reported from Surtsey before (Bryum elegans, Ceratodon heterophyllus, Didymodon rigidulus, Eurhynchium praelongum, Schistidium confertum, S. papillosum, Tortula hoppeana and T. muralis). Habitat loss due to erosion and reduced thermal activity in combination with successional vegetation changes are likely to have played a significant role in the decline of some bryophyte species which were abundant in 1972 (Leptobryum pyriforme, Schistidium apocarpum coll., Funaria hygrometrica, Philonotis spp., Pohlia spp, Schistidium strictum, Sanionia uncinata) while others have continued to thrive and expand (e.g. Schistidium maritimum, Racomitrium lanuginosum, R. ericoides, $R$. fasciculare and Bryum argenteum). Some species (especially Bryum spp.) benefit from the formation of new habitats, such as grassland within a gull colony, which was established in 1984. Several newcomers are rarely producing
\end{abstract}

sporophytes on Iceland and are unlikely to have been dispersed by airborne spores. They are more likely to have been introduced to Surtsey by seagulls in the form of vegetative fragments or dispersal agents (Bryum elegans, Didymodon rigidulus, Eurhynchium praelongum, Ceratodon heterophyllus and Ulota phyllantha). The establishment of the gull colony also means that leakage of nutrients from the nesting area is, at least locally, downplaying the relative importance of nitrogen fixation by cyanobacteria growing in bryophyte shoots.

\section{Introduction}

The volcanic island Surtsey $\left(63^{\circ} \mathrm{N}, 20^{\circ} \mathrm{W}\right)$ surfaced in November 1963 (Pórarinsson, 1965) in an eruption that lasted until June 1967. It belongs to Vestmannaeyjar, an archipelago of volcanic origin, $7-33 \mathrm{~km}$ off the south coast of Iceland (Magnússon et al., 2009). The area of the island reached $2.65 \mathrm{~km}^{2}$ by the end of the eruption (Jakobsson et al., 2000). In the early years the erosion of the island was relatively fast and even though the island is now shrinking at a slower pace the erosion is likely to continue until the palagonite core has been reached. In 1998 the area of Surtsey was down to $1.47 \mathrm{~km}^{2}$ (Jakobsson et al., 2000), in 2004 it was $1.41 \mathrm{~km}^{2}$ (Jakobsson et al., 2007) and it has been estimated that only $0.5 \mathrm{~km}^{2}$ of the island will remain by 2100 (Jakobsson and Guðmundsson, 2003). 


\subsection{Bryophyte succession on volcanic land}

Bryophytes are well known as primary colonizers on volcanic land. They produce organic material that may later serve as substrate for secondary colonists. They are also indirectly responsible for a small but significant input of nitrogen to the ecosystem, which is produced by cyanobacteria associated with the moss shoots (Henriksson et al., 1987). In comparison, free-living cyanobacteria and non-photosynthetic nitrogen-fixing bacteria (Azotobacter) appear to supply insignificant amounts of nitrogen (Henriksson et al., 1987), as observed in the early phase of the Surtsey colonization (Henriksson and Henriksson, 1982). Bryophytes are known to play a significant role in plant succession in Iceland, Japan and Italy (Tagawa et al., 1985) but seem to be less important in the Tropics as exemplified by studies from Mt. Cameroon in Africa and Krakatau Islands in Indonesia (Fonge et al., 2005; Penzig, 1902 in Whittaker et al., 1984). In Hawaii, species of Campylopus are important in lava rock succession and often found on rocks otherwise devoid of plants. Likewise, Grimmia trichophylla and Racomitrium lanuginosum, are found on more exposed lava and, as an example, were the only plant species found above $2440 \mathrm{~m}$ a.s.l. on lava flows of Mauna Loa volcano in Hawaii (Miller, 1960). In Icelandic lava fields, $R$. lanuginosum is one of the most abundant plant species and often forms vast and thick carpets where conditions are favourable (e.g. Bjarnason, 1991; Jóhannsson, 1993).

\subsection{Bryophyte colonization on Surtsey}

Volcanic ash samples were taken on a transect on Surtsey in June 1967, and cultured in a search for freshwater biota. Nothing grew from those cultures except moss protonema (Maguire Jr., 1968). Only two months later, in early August 1967, the first moss mats made up of Funaria hygrometrica were found on the island, growing on a sand bank at the edge of a lagoon. Patches of moss growth were later that year found at the edge of a lava crater in the central part of the island. Those patches were made up of Funaria hygrometrica and Bryum argenteum (Jóhannsson, 1968). In 1968, a total of seven moss species were found (excluding unidentified Bryum spp.): Dicranella crispa, Leptobryum pyriforme, Pohlia bulbifera, P. cruda, Bryum argenteum, Funaria hygrometrica and Ceratodon purpureus (Friðriksson, 1970). In the subsequent year (1969) the moss layer became more conspicuous, possibly because of frequent summer rains. That summer, eight moss species were found: Bryum argentum, $B$. caespiticium, B. capillare, Dicranella crispa, Funaria hygrometrica, Leptobryum pyriforme, Pogonatum urnigerum and Racomitrium canescens - four of which were new for the island (Table 1) (Friðriksson and Johnsen, 1968; Friðriksson et al., 1972a).

In the summer of 1970, a special effort to search for moss patches was made in most parts of the lava fields in the southern section of the island, which revealed that the moss colonies were still small and scattered. A considerable expansion was observed compared to the previous years. A total of 17 species were found excluding two taxa that could only be determined to genus level. Funaria hygrometrica and Racomitrium canescens, together with Bryum spp. were dominants, whereas the less abundant species were restricted to specific microhabitats. Three species were found with spore capsules: Funaria hygrometrica, Ceratodon purpureus and Dicranella crispa (Table 1) (Friðriksson et al., 1972a).

A more intensive and systematic survey of bryophytes was done for the first time in 1971. A grid system developed in 1968 which divided the island into $100 \mathrm{~m} \times 100 \mathrm{~m}$ quadrats (Friðriksson and Johnsen, 1968) was used and each quadrat was investigated separately. The same survey was repeated the following year (1972), now also including the registration of habitat choice and habitat abundance for each species. In 1971, 36 bryophyte species were found, of which 20 were new for the island (Table 1), including the first liverwort species, Marchantia polymorpha. All the new species were rare except Bryum stenotrichum. As a consequence of the second survey the total number of species increased from 36 in 1971 to 68 in 1972 (Table 1). Just as in the previous year, all the new species recorded in 1972 were rare. Three liverworts were among these new species but they were only assigned to genera. On the other hand three species were not rediscovered and it was concluded that at least one, Fissidens adianthoides, had become extinct since it was not recovered at the original location. One of the new species, Bryum klinggraeffii was unknown from Iceland at the time (Magnússon and Friðriksson, 1974) but has later been recorded at several locations in geothermal areas (Jóhannsson, 1995).

In the herbaria (ICEL and AMNH) of the Icelandic Institute of Natural History, bryophyte specimens from Surtsey have been deposited from the years 1975, 1976, 1984, 1990, 1991, 2003 and 2006 in addition to the years 1967-1972 (Table 1), but systematic bryophyte inventories are lacking during the time span between 1972 and 2008, when the inventory presented here was carried out. To the best of our knowledge no reports relating to bryophyte species on Surtsey have been published since 2009 (Magnússon et al., 2009).

The aim of our study was to get a picture of the present distribution of bryophyte species on Surtsey and to update the information on the status of the bryophyte communities. We also wanted to summarize the successional changes that have occurred during the 50 years since the island emerged from the sea and the 40 years since the last bryophyte survey.

\section{Material and methods}

Surtsey was visited for bryophyte collection during 7-10 July 2008 by GVI. For sampling, the same $100 \mathrm{~m} \times 100 \mathrm{~m}$ grid (Fig. 1) (Friðriksson and Johnsen, 1968) was used as 


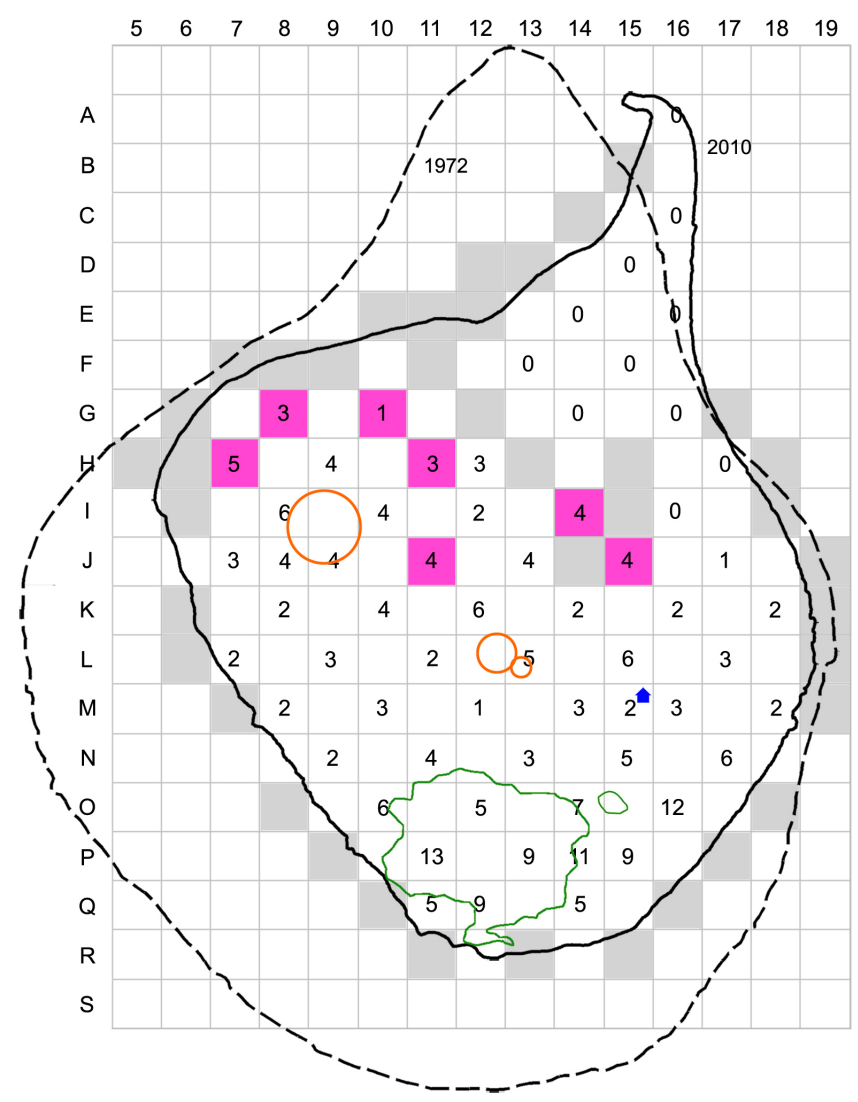

Figure 1. Number of bryophyte species found in each searched quadrat $(100 \mathrm{~m} \times 100 \mathrm{~m})$ in 2008 . Quadrats filled with light grey colour were deemed inaccessible. Quadrats filled with pink colour had visible steam emission. Orange circles indicate the craters and green polygons show where there is dense vegetation and a colony of seagulls. The blue house indicates the location of the research facilities and helicopter platform (M15). Maps: Anette Meier and GVI.

in the earlier surveys of bryophyte distribution on Surtsey in 1970-1972 (Friðriksson et al., 1972a; Magnússon and Friðriksson, 1974). Every other $100 \mathrm{~m} \times 100 \mathrm{~m}$ quadrat of the island was searched and sampled as long as it was relatively easily accessible; five additional quadrats were sampled for the following reasons: (1) to make up for the lack of sampling in an inaccessible quadrat (H12 instead of H13); (2) to complete the inventory in cases where a certain quadrat seemed likely to reveal additional species not found elsewhere (J8 and P14); (3) to see whether the presence of the research facilities and helicopter platform had any notable effect on species composition (M15); and (4) by mistake when the first quadrat to be visited was Q11 albeit it was supposed to be Q12. The sampled quadrats will hereafter be referred to as the selected quadrats, which were thus studied in all three systematic inventories (1971, 1972 and 2008; Fig. 1).

To establish the position of each square a handheld Garmin GPS was used with an accuracy of about $\pm 10 \mathrm{~m}$. An ef- fort was made to avoid samples with dubious assignment to squares, that is, sampling at the border between quadrats was avoided, unless a particular species was absent from inside a quadrat and only present along the borders. The bryophytes were put in paper bags and dried for later identification. The nomenclature follows the latest checklist for European mosses (Burley and Pritchard, 1990; Hill et al., 2006) and the latest official checklist for Icelandic liverworts (Jóhannsson, 2003). The samples are stored at the Icelandic Institute of Natural History for future reference. Information from specimens in the herbaria (ICEL and AMNH) of the Icelandic Institute of Natural History is also compiled here (Table 1).

\section{Results and discussion}

\subsection{Bryophyte distribution}

In total, 63 quadrats were screened $(100 \mathrm{~m} \times 100 \mathrm{~m}$ each). In 11 of these no bryophytes were observed (Fig. 1). All the quadrats lacking bryophytes were located in the northern, lower part of the island which was mostly made up of sand and boulders. The region where Surtsey is located is exposed to strong disturbance by wind and wave action. Due to the wrath of the elements, the porous lava fields, mostly in the south and southwestern part of Surtsey, have been eroding relatively fast and will continue to do so in the future (Fig. 1) (Jakobsson and Guðmundsson, 2003). Between 1972 and 2008 at least 87 quadrats of land (37 with bryophyte cover) have been lost to the sea, leaving more sandy lava fields compared to those that have been lost. Nevertheless, bryophyte distribution has expanded among the remaining quadrats so that bryophytes were found in nine quadrats that were reported to be empty in 1972 .

\subsection{Species richness}

The most species-rich areas were in the southern part of Surtsey which also had the densest vegetation and a teeming seagull colony stretching over 10 ha of land (Magnússon et al., 2009). A maximum number of 13 bryophyte species was found in quadrat $\mathrm{P} 11$; the second highest number of species was found in quadrat $\mathrm{O} 16$ with 12 species; 11 species were found in P14; and 9 species in Q12, P13 and P15 respectively. All these quadrats were in the southernmost part of Surtsey, within or around the gull colony (Fig. 1).

Quadrat M15 was included in order to see whether the presence of the research facilities and the helicopter platform had some obvious effect on species richness; that turned out not to be the case since only two bryophyte species were recorded there: Racomitrium lanuginosum and Schistidium maritimum, both common on the island.

The three genera Bryum, Racomitrium and Schistidium were especially influential in terms of species numbers and abundance on Surtsey. Numerous unidentifiable specimens of Bryum and Schistidium were collected in 2008, whereas all 

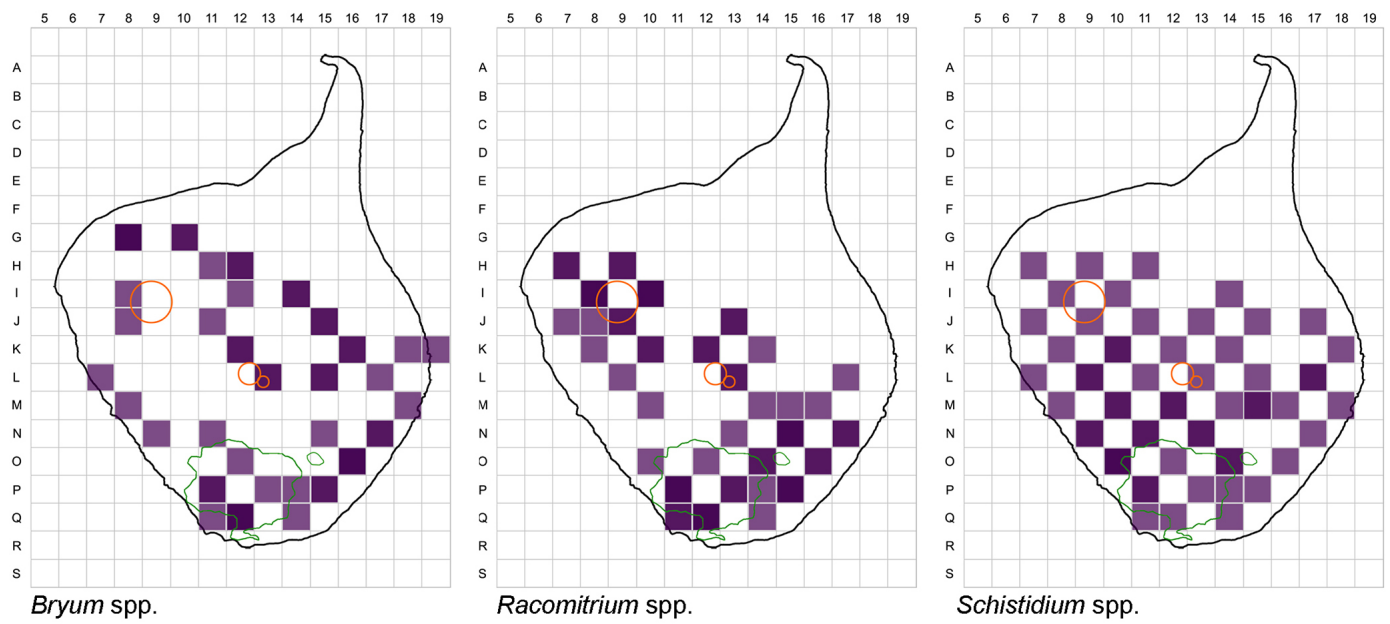

Figure 2. Distribution of the most common genera on Surtsey: Bryum, Racomitrium and Schistidium. Each species or taxa layer has $70 \%$ colour opacity; darker colour indicates more species and lighter colour fewer species. Maps: Anette Meier and GVI.

specimens of Racomitrium were identified to species level. For the genus Bryum, which is renowned for being difficult to identify to species level (e.g. Jóhannsson, 1995), in total 13 species have been identified through the years, making it the most species-rich bryophyte genus on Surtsey. In the 2008 inventory only four species were able to be identified for certain (Table 1) but specimens of the genus were found in 33 quadrats - thereof unidentified in 26 quadrats (Fig. 2). Grimmiaceae (represented by Schistidium and Racomitrium) is by far the most widespread and frequent family of bryophytes on Surtsey, which can be explained by the fact that representatives of this family commonly act as primary colonists on bare and exposed rocks. Members of Grimmiaceae in general constitute important components of the vegetation of Icelandic lava fields (e.g. Bjarnason, 1991; Frey et al., 2006; Jóhannsson, 1993). For the genus Schistidium seven species have been encountered on Surtsey. During the 2008 inventory five species were identified and the genus was found in 45 quadrats. Four species of Racomitrium were found in 33 quadrats in 2008 out of five species earlier observed (Table 1).

By 1969 altogether 21 species had been found on Surtsey and all but three of them (Bryum caespiticium, B. capillare and Pohlia bulbifera) were rediscovered 1970 (note that $B$. capillare was however present in 2008; Table 1). All 18 bryophyte species discovered in 1970 (Friðriksson et al., 1972b) were then again present on the island in 1972 but only seven of them were found in 2008 (Fig. 3). A comparison between the surveys from 1972 and 2008 suggests fairly strong changes in bryophyte species diversity -68 species were found in 1972 and only 38 species in 2008 . The data also point to a strong turnover: 18 species found in 2008 were new to the island, whereas 51 species found in 1972 were not observed in 2008. Some of these species might very well still occur on Surtsey since the 2008 survey was limited to in-

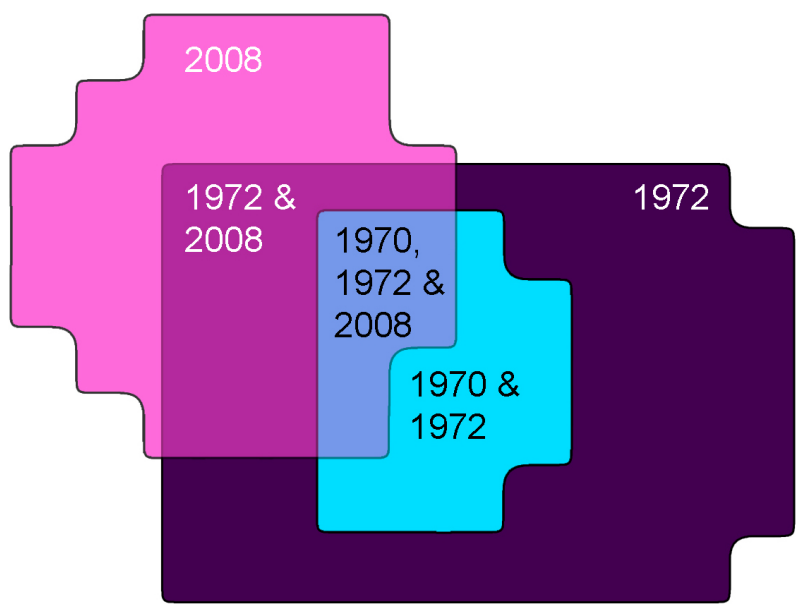

Figure 3. Area proportional Euler diagram for the total number of species found on Surtsey in 1970, 1972 and 2008. Each polygon indicates a total species number for a given year: $1970=17,1972=$ $72,2008=38$; the intersections indicate the species in common.

clude only every second quadrat. Small species are also easy to overlook even if they were present in the selected quadrats. The distribution of the most common species amongst these 51 should be considered to have greatly decreased in abundance. It is however impossible to draw any certain conclusions regarding the rare species.

Apart from this, it is clear that decades of erosion have led to loss of significant parts of the island and profound effects on the remaining areas. Since changes in distribution and abundance of the rare species may be difficult to interpret, we restrict the following discussion to changes among the dominant species. 

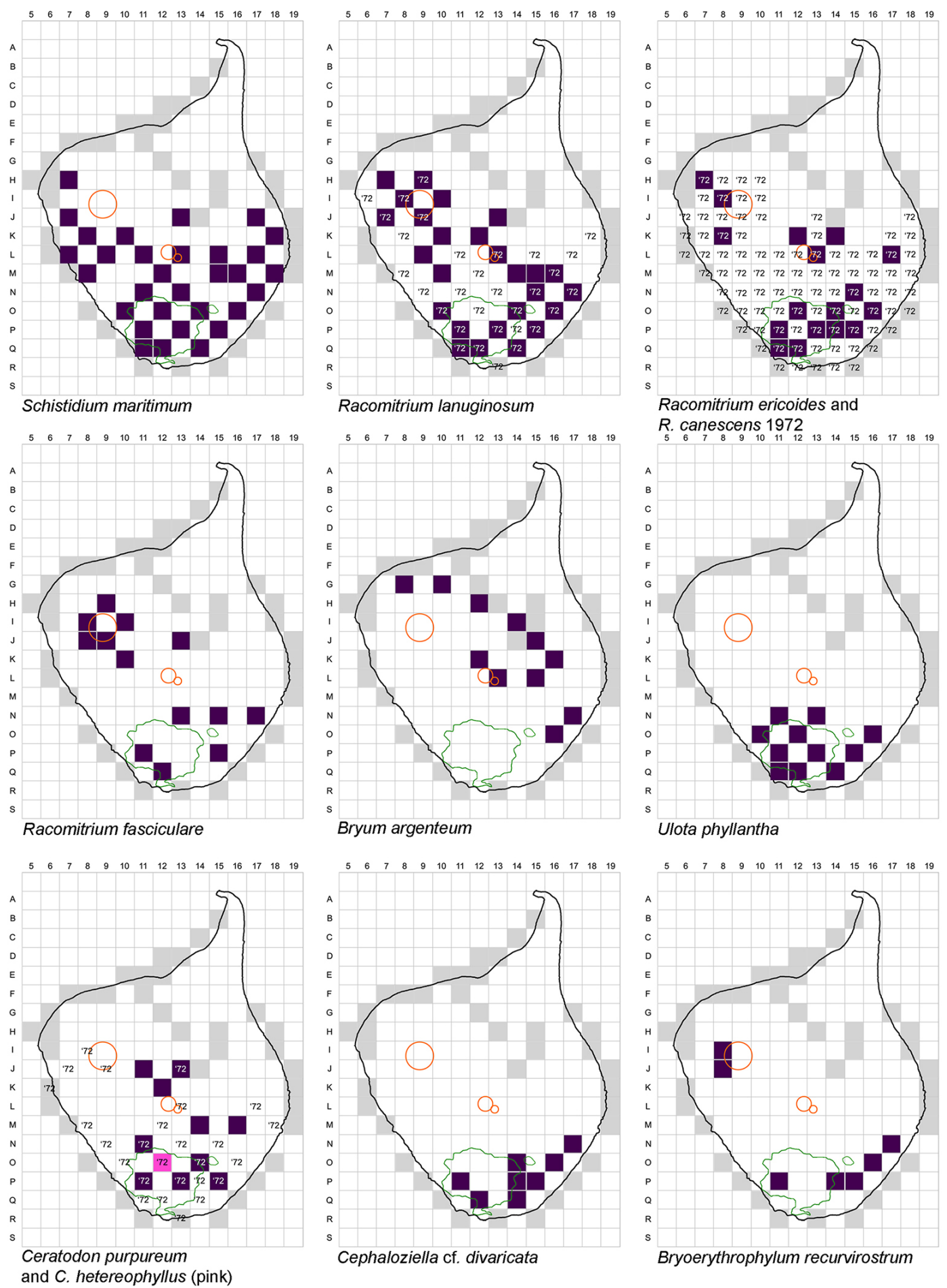

Figure 4. Distribution maps for the most widespread bryophyte species found in 2008, compared with distribution in 1972 when applicable. Ceratodon heterophyllus is included in a different colour (pink). Orange circles indicate the craters and the green contours indicate the seagull colony area with dense vegetation. Grey quadrats indicate inaccessible areas; the contour of Surtsey is from 2010; each quadrat is $100 \mathrm{~m} \times 100 \mathrm{~m}$. Maps: Anette Meier and GVI. 
Table 1. List of all bryophyte species found on Surtsey since the birth of the island. Note that specimens of $R$. canescens present in ICEL are identified as $R$. canescens var. ericoides but apparently published simply as $R$. canescens. $¥$ : No herbarium specimens; Bold $\times$ : herbarium specimen in ICEL in addition to being mentioned in the main reference; A: Jóhannsson, 1968; B: Friðriksson, 1970; C: Bjarnason and Friðriksson, 1972; D: Friðriksson et al., 1972a; E: Magnússon and Friðriksson, 1974; F: Magnússon and Friðriksson, 1974; G: Magnússon and Magnússon, 2003, unpublished data; H: Own data; I: Friðriksson et al., 1972b.

\begin{tabular}{|c|c|c|c|c|c|c|c|c|c|c|c|c|c|c|c|c|}
\hline & $\begin{array}{l}\text { Main reference: } \\
\text { Year: }\end{array}$ & $\begin{array}{c}\text { A } \\
1967\end{array}$ & $\begin{array}{c}\text { B } \\
1968\end{array}$ & $\begin{array}{c}\text { C } \\
1969\end{array}$ & $\begin{array}{c}\mathrm{D} \\
1970\end{array}$ & $\begin{array}{c}E \\
1971\end{array}$ & $\begin{array}{c}F \\
1972\end{array}$ & $\begin{array}{r}\text { ICEL } \\
1975\end{array}$ & $\begin{array}{r}\text { ICEL } \\
1976\end{array}$ & $\begin{array}{r}\text { ICEL } \\
1984\end{array}$ & $\begin{array}{l}\text { ICEL } \\
1990\end{array}$ & $\begin{array}{r}\text { ICEL } \\
1991\end{array}$ & $\begin{array}{c}\mathrm{G} \\
2003\end{array}$ & $\begin{array}{l}\text { AMNH } \\
2006\end{array}$ & $\begin{array}{c}\mathrm{H} \\
2008\end{array}$ & $\begin{array}{c}\text { Number } \\
\text { of occur- } \\
\text { rences }\end{array}$ \\
\hline \multicolumn{17}{|c|}{ Mosses } \\
\hline & Bryum argenteum Hedw. & $\mathrm{x}$ & $\times$ & $\times$ & $\times$ & $\mathbf{x}$ & $\times$ & & & $\mathrm{x}$ & $x$ & & $\mathbf{x}$ & & $\times$ & 10 \\
\hline & Funaria hygrometrica Hedw. & $\mathbf{x}$ & $x$ & $x$ & $\mathrm{x}$ & $x$ & $x$ & & & $\mathrm{x}$ & & & & & $x$ & 8 \\
\hline & Bryum spp. & & I & I & $x$ & $x$ & $x$ & & & & & & $x$ & & $x$ & 7 \\
\hline & Ceratodon purpureus (Hedw.) Brid. & & $x$ & & $x$ & $x$ & $x$ & $\mathbf{x}$ & & $\mathbf{x}$ & $x$ & & $x$ & & $x$ & 9 \\
\hline & Dicranella crispa (Hedw.) Schimp. & & I & I & $\mathbf{x}$ & $\mathbf{x}$ & $x$ & & & & $\mathbf{x}$ & & & & & 6 \\
\hline & Leptobryum pyriforme (Hedw.) Wilson & & $x$ & $x$ & $\mathrm{x}$ & $x$ & $x$ & & & $\mathbf{x}$ & & & & & & 6 \\
\hline & Pohlia bulbifera (Warnst.) Warnst. & & $x$ & & & & & & & & & & & & & 1 \\
\hline & Pohlia cruda (Hedw.) Lindb. & & I & & $x$ & $\times$ & $x$ & $\mathrm{x}$ & & $\mathbf{x}$ & $x$ & & & & & 7 \\
\hline$¥$ & Bryum caespiticium Hedw. & & & $\times$ & & & & & & & & & & & & 1 \\
\hline \multirow[t]{15}{*}{$¥$} & Bryum capillare Hedw. & & & $\times$ & & & & & & & & & & & $\times$ & 2 \\
\hline & Pogonatum urnigerum (Hedw.) P. Beauv. & & & $x$ & $\mathbf{x}$ & $\times$ & $x$ & & & $\mathbf{x}$ & & & & & $x$ & 6 \\
\hline & Racomitrium canescens (Hedw.) Brid. & & & $\times$ & $x$ & $\times$ & $\times$ & & & & & & & & & 4 \\
\hline & Aongstroemia longipes (Sommerf.) Bruch \& Schimp. & & & & $\mathrm{x}$ & & $\mathrm{x}$ & & & & & & & & & 2 \\
\hline & Atrichum undulatum (Hedw.) P. Beauv. & & & & & $\times$ & $\mathrm{x}$ & & & & $x$ & & & & & 4 \\
\hline & $\begin{array}{l}\text { Brachythecium salebrosum (Hoffm. ex F. Weber } \\
\text { \& D. Mohr) Schimp. }\end{array}$ & & & & $\mathbf{x}$ & & $\times$ & $x$ & & & & & $x$ & & & 4 \\
\hline & Bryum dichotomum Hedw. & & & & ICEL & & & $\mathbf{x}$ & & $\mathbf{x}$ & $\mathbf{x}$ & & $\mathbf{x}$ & & $x$ & 6 \\
\hline & Bryum pallens Sw. ex anon. & & & & $\mathbf{x}$ & $x$ & $x$ & & & $\mathbf{x}$ & & & & & & 4 \\
\hline & Dichodontium pellucidum (Hedw.) Schimp. & & & & $\mathbf{x}$ & $x$ & $x$ & $\mathbf{x}$ & & $\mathbf{x}$ & & & & & $x$ & 6 \\
\hline & Philonotis spp. & & & & $x$ & $x$ & $x$ & & & & & & & & & 3 \\
\hline & Pohlia wahlenbergii (F. Weber \& D. Mohr) A. L. Andrews & & & & $\mathbf{x}$ & $x$ & $\times$ & & & $\mathbf{x}$ & & & & & & 4 \\
\hline & Racomitrium lanuginosum (Hedw.) Brid. & & & & $\mathbf{x}$ & $x$ & $x$ & & $\mathrm{x}$ & $\mathbf{x}$ & $\mathbf{x}$ & & $x$ & & $x$ & 8 \\
\hline & Sanionia uncinata (Hedw.) Loeske & & & & $x$ & $x$ & $\times$ & & & $\mathbf{x}$ & $\mathbf{x}$ & & $x$ & & $x$ & 7 \\
\hline & Anomobryum julaceum (Schrad. ex P. Gaertn. et al.) Schimp. & & & & & $x$ & $x$ & & & $\mathbf{x}$ & & & & & & 3 \\
\hline & Bartramia ithyphylla Brid. & & & & & $x$ & $\times$ & & & $x$ & & & & & & 3 \\
\hline & Bryoerythrophyllum recurvirostrum (Hedw.) P. C. Chen & & & & & $\mathrm{x}$ & $\times$ & & & $\mathbf{x}$ & $\mathrm{x}$ & & & & $\times$ & 5 \\
\hline \multirow[t]{2}{*}{$¥$} & Bryum algovicum Sendtn. ex Müll.Hal. & & & & & $x$ & $x$ & & & & & & & & & 2 \\
\hline & Bryum archangelicum Bruch \& Schimp. & & & & & $x$ & $x$ & $\mathrm{x}$ & & $\mathbf{x}$ & $\mathbf{x}$ & & & & & 5 \\
\hline$¥$ & Bryum arcticum (R.Br.) Bruch \& Schimp. & & & & & $x$ & $x$ & & & & & & & & & 2 \\
\hline$¥$ & Dicranella schreberiana (Hedw.) Dixon & & & & & $x$ & & & & & & & & & & 1 \\
\hline & Dicranella varia (Hedw.) Schimp. & & & & & $x$ & $\mathbf{x}$ & & & & $x$ & & & & & 3 \\
\hline & Distichium capillaceum (Hedw.) Bruch et Schimp. & & & & & $x$ & $x$ & & & $\mathbf{x}$ & $\mathbf{x}$ & & & & & 4 \\
\hline & Drepanocladus polygamus (Schimp.) Hedenäs & & & & & $\mathrm{x}$ & $x$ & & & $\mathrm{x}$ & $\mathrm{x}$ & & & & & 4 \\
\hline$¥$ & Encalypta ciliata Hedw. & & & & & $x$ & $\times$ & & & & & & & & & 2 \\
\hline$¥$ & Fissidens adianthoides Hedw. & & & & & $x$ & & & & & & & & & & 1 \\
\hline & Mnium hornum Hedw. & & & & & $x$ & $\times$ & & & & $\mathbf{x}$ & & & & $x$ & 4 \\
\hline$¥$ & Oncophorus virens (Hedw.) Brid. & & & & & $x$ & $\times$ & & & & & & & & & 2 \\
\hline$¥$ & Pohlia annotina (Hedw.) Lindb. & & & & & $x$ & & & & & & & & & & 1 \\
\hline$¥$ & Polytrichastrum alpinum (Hedw.) G. L. Sm. & & & & & $x$ & $x$ & & & & & & & & & 2 \\
\hline$¥$ & Polytrichastrum longisetum (Sw. ex Brid.) G. L. Sm. & & & & & $\times$ & $\times$ & & & & & & & & & 2 \\
\hline$¥$ & Rhytidiadelphus squarrosus (Hedw.) Warnst. & & & & & $x$ & $x$ & & & & & & & & $x$ & 3 \\
\hline$¥$ & Schistidium apocarpum (Hedw.) Bruch \& Schimp. & & & & & $x$ & $x$ & & & & & & & & & 2 \\
\hline & Schistidium maritimum (Sm. ex R.Scott) Bruch \& Schimp. & & & & & $x$ & $\times$ & & & $\mathbf{x}$ & $\mathrm{x}$ & & $\mathbf{x}$ & $x$ & $x$ & 7 \\
\hline$¥$ & Straminergon stramineum (Dicks. ex Brid.) Hedenäs & & & & & $x$ & $x$ & & & & & & & & & 2 \\
\hline$¥$ & Marantia polymorpha $\mathrm{L}$. & & & & & $x$ & $x$ & & & & & & & & & 2 \\
\hline & Amblystegium serpens (Hedw.) Schimp. & & & & & & $x$ & & & & $\mathbf{x}$ & $\mathrm{x}$ & & & & 3 \\
\hline$¥$ & Amphidium lapponicum (Hedw.) Schimp. & & & & & & $x$ & & & & & & & & & 1 \\
\hline$¥$ & Aulacomnium palustre (Hedw.) Schwägr. & & & & & & $x$ & & & & & & & & & 1 \\
\hline & Barbula unguiculata Hedw. & & & & & & $x$ & & & $\mathrm{x}$ & & & & & $\times$ & 3 \\
\hline & Brachythecium albicans (Hedw.) Schimp. & & & & & & $x$ & & & & & & $\mathrm{x}$ & & $x$ & 3 \\
\hline$¥$ & Brachythecium rivulare Schimp. & & & & & & $x$ & & & & & & & & & 1 \\
\hline$¥$ & Bryum calophyllum $\mathrm{R} . \mathrm{Br}$. & & & & & & $x$ & & & & & & & & & 1 \\
\hline & Bryum klinggraeffii Schimp. & & & & & & $\mathbf{x}$ & & & & & & & & & 1 \\
\hline & Bryum pallescens Schleich. ex Schwägr. & & & & & & $x$ & & & $\mathrm{x}$ & $\mathrm{x}$ & & & & & 3 \\
\hline$¥$ & Calliergonella lindbergii (Mitt.) Hedenäs & & & & & & $\times$ & & & & & & & & & 1 \\
\hline$¥$ & Dicranella heteromalla (Hedw.) Schimp. & & & & & & $x$ & & & & & & & & & 1 \\
\hline$¥$ & Dicranella subulata (Hedw.) Schimp. & & & & & & $x$ & & & & & & & & & 1 \\
\hline & Dicranoweisia crispula (Hedw.) Milde & & & & & & $x$ & & & $\mathbf{x}$ & & & & & $\times$ & 3 \\
\hline$¥$ & Didymodon brachyphyllus (Sull.) R.H.Zander & & & & & & $x$ & & & & & & & & $x$ & 2 \\
\hline & Didymodon fallax (Hedw.) R. H. Zander & & & & & & $x$ & & & $\mathbf{x}$ & & & & & $\times$ & 3 \\
\hline$¥$ & Didymodon icmadophilus (Schimp. ex Müll.Hal.) K.Saito & & & & & & $x$ & & & & & & & & & 1 \\
\hline & Ditrichum heteromallum (Hedw.) E. Britton & & & & & & $x$ & $\mathrm{x}$ & & $\mathrm{x}$ & & & & & & 3 \\
\hline$¥$ & Drepanocladus aduncus (Hedw.) Warnst. & & & & & & $x$ & & & & & & $x$ & & & 2 \\
\hline & Encalypta sp. & & & & & & $x$ & & & & & & & & & 1 \\
\hline$¥$ & Grimmia torquata Drumm. & & & & & & $x$ & & & & & & & & & 1 \\
\hline$¥$ & Isopterygiopsis pulchella (Hedw.) Z. Iwats. & & & & & & $x$ & & & & & & & & & 1 \\
\hline$¥$ & Philonotis fontana (Hedw.) Brid. & & & & & & $x$ & & & & & & & & $x$ & 2 \\
\hline$¥$ & Plagiomnium cuspidatum (Hedw.) T. J. Kop. & & & & & & $\times$ & & & & & & & & & 1 \\
\hline$¥$ & Pohlia filum (Schimp.) Mårtensson & & & & & & $x$ & & & & & & & & & 1 \\
\hline$¥$ & Pohlia proligera $($ Kindb.) Lindb. ex Broth. & & & & & & $\times$ & & & & & & & & & 1 \\
\hline$¥$ & Polytrichastrum sphaerothecium (Besch.) J.-P. Frahm & & & & & & $\times$ & & & & & & & & & 1 \\
\hline$¥$ & Polytrichum piliferum Hedw. & & & & & & $x$ & & & & & & & & & 1 \\
\hline$¥$ & Psilopilum laevigatum (Wahlenb.) Lindb. & & & & & & $\times$ & & & & & & & & & 1 \\
\hline$¥$ & Racomitrium sudeticum (Funck) Bruch \& Schimp. & & & & & & $x$ & & & & & & & & $\times$ & 2 \\
\hline
\end{tabular}


Table 1. Continued.

\begin{tabular}{|c|c|c|c|c|c|c|c|c|c|c|c|c|c|c|c|c|}
\hline & $\begin{array}{l}\text { Main reference: } \\
\text { Year: }\end{array}$ & $\begin{array}{c}\text { A } \\
1967\end{array}$ & $\begin{array}{c}\text { B } \\
1968\end{array}$ & $\begin{array}{c}C \\
1969\end{array}$ & $\begin{array}{c}\mathrm{D} \\
1970\end{array}$ & $\begin{array}{c}E \\
1971\end{array}$ & $\begin{array}{c}F \\
1972\end{array}$ & $\begin{array}{r}\text { ICEL } \\
1975\end{array}$ & $\begin{array}{r}\text { ICEL } \\
1976\end{array}$ & $\begin{array}{r}\text { ICEL } \\
1984\end{array}$ & $\begin{array}{r}\text { ICEL } \\
1990\end{array}$ & $\begin{array}{r}\text { ICEL } \\
1991\end{array}$ & $\begin{array}{c}\mathrm{G} \\
2003\end{array}$ & $\begin{array}{l}\text { AMNH } \\
2006\end{array}$ & $\begin{array}{c}\mathrm{H} \\
2008\end{array}$ & $\begin{array}{c}\text { Number } \\
\text { of occur- } \\
\text { rences }\end{array}$ \\
\hline \multicolumn{17}{|c|}{ Mosses } \\
\hline & Trichodon cylindricus (Hedw.) Schimp & & & & & & $x$ & & & & & & & & & 1 \\
\hline \multirow[t]{19}{*}{$¥$} & Trichostomum brachydontium Bruch & & & & & & $\times$ & & & & & & & & $\times$ & 2 \\
\hline & Cephaloziella divaricata $(\mathrm{Sm}$.$) Schiffn.$ & & & & & & ICEL & & & & & & $\mathbf{x}$ & $x$ & cf. & 4 \\
\hline & Cephaloziella spp. & & & & & & $\times$ & & & & & & & & & 1 \\
\hline & Jungermannia sp. (atrovirens or pumila) & & & & & & $\times$ & & & & & & & & & 1 \\
\hline & Scapania sp. (curta or scandica) & & & & & & $\times$ & & & & & & & & $x$ & 2 \\
\hline & Philonotis arnellii Husn. & & & & & & & $\mathbf{x}$ & & $\mathrm{x}$ & & & & & & 2 \\
\hline & Racomitrium fasciculare (Hedw.) Brid. & & & & & & & $\mathbf{x}$ & & $\mathbf{x}$ & $\mathbf{x}$ & & $x$ & & $x$ & 5 \\
\hline & Reboulia hemisphaerica (L.) Raddi & & & & & & & $\mathbf{x}$ & & & & & & & $\times$ & 2 \\
\hline & Brachytheciastrum velutinum (Hedw.) Ignatov \& Huttunen & & & & & & & & & $x$ & & & & & & 1 \\
\hline & Didymodon insulanus (De Not.) M. O. Hill & & & & & & & & & $\mathbf{x}$ & $\mathbf{x}$ & & & & & 2 \\
\hline & Racomitrium ericoides (Brid.) Brid. & & & & ICEL & & & & & $x$ & $\mathbf{x}$ & & $x$ & & $x$ & 4 \\
\hline & Schistidium strictum (Turner) Loeske ex Mårtensson & & & & & & & & & $x$ & $\mathbf{x}$ & & $\times$ & & $\times$ & 4 \\
\hline & Cephaloziella hampeana (Nees) Schiffn. & & & & & & & & & $\mathbf{x}$ & & & $\times$ & & cf. & 3 \\
\hline & Jungermannia pumila With. & & & & & & & & & $\mathrm{x}$ & & & & & & 1 \\
\hline & Lophozia excisa (Dicks.) Dumort. & & & & & & & & & $\mathbf{x}$ & & & & & & 1 \\
\hline & Brachythecium rutabulum (Hedw.) Schimp. & & & & & & & & & & $\mathbf{x}$ & & & & & 1 \\
\hline & Philonotis tomentella Molendo & & & & & & & & & & $\mathbf{x}$ & & & & & 1 \\
\hline & Schistidium frigidum $\mathrm{H}$. H. Blom & & & & & & & & & & $\mathbf{x}$ & & & & & 1 \\
\hline & Bryum salinum I. Hagen ex Limpr. & & & & & & & & & & & & ICEL & & & 1 \\
\hline \multirow[t]{4}{*}{$¥$} & Schistidium flexipile (Lindb. ex Broth.) G. Roth & & & & & & & & & & & & $\times$ & & $x$ & 2 \\
\hline & Ulota phyllantha Brid. & & & & & & & & & & & & $x$ & & $x$ & 2 \\
\hline & Brachythecium sp. & & & & & & & & & & & & & & $\times$ & 1 \\
\hline & Bryum elegans Nees & & & & & & & & & & & & & & $\times$ & 1 \\
\hline$¥$ & Ceratodon heterophyllus Kindb. & & & & & & & & & & & & & & cf. & 1 \\
\hline \multirow[t]{2}{*}{$¥$} & Didymodon rigidulus Hedw. & & & & & & & & & & & & & & $x$ & 1 \\
\hline & Didymodon sp. & & & & & & & & & & & & & & $x$ & 1 \\
\hline \multirow[t]{2}{*}{$¥$} & Kindbergia praelonga (Hedw.) Ochyra & & & & & & & & & & & & & & $x$ & 1 \\
\hline & Pohlia sp. & & & & & & & & & & & & & & $x$ & 1 \\
\hline$¥$ & Schistidium confertum (Funck) Bruch \& Schimp. & & & & & & & & & & & & & & $x$ & 1 \\
\hline \multirow[t]{2}{*}{$¥$} & Schistidium papillosum Culm. & & & & & & & & & & & & & & $x$ & 1 \\
\hline & Schistidium sp. & & & & & & & & & & & & & & $x$ & 1 \\
\hline$¥$ & Tortula hoppeana (Schultz) Ochyra & & & & & & & & & & & & & & $\times$ & 1 \\
\hline \multirow[t]{2}{*}{$¥$} & Tortula muralis Hedw. & & & & & & & & & & & & & & $\times$ & 1 \\
\hline & Total number of taxa: & 2 & 8 & 9 & 19 & 38 & 72 & 10 & 1 & 33 & 24 & 1 & 18 & 2 & 43 & \\
\hline
\end{tabular}

\subsection{Early colonizers}

The most widespread bryophyte species on Surtsey in 2008 was Schistidium maritimum (observed in 31 quadrats; Fig. 4). This species grows on rocks near the sea all around Iceland (also in Vestmannaeyjar), although most common in west Iceland. It usually produces spore capsules, both on the Icelandic mainland (Jóhannsson, 1993) and on Surtsey. It was first encountered in 1971 but has since then been found in 1972, 1984, 1990, 2003, 2006 and now 2008 (Table 1).

Racomitrium lanuginosum was abundant both in 1972 (33 of the selected quadrats) and in 2008 (26 quadrats, of which eight were previously unreported; Fig. 4). This species can be considered prominent and easily distinguishable and therefore unlikely to have been overlooked in the earlier surveys. It was the second most widespread species in 2008 and without doubt the most abundant bryophyte species. It formed a thick carpet in the westernmost and largest crater, Surtungur (Fig. 5). This species had already been found on Surtsey in 1970. It is extremely common and abundant on the Icelandic mainland (also in Vestmannaeyjar) where it is a characteristic component of the vegetation of lava fields (e.g. Bjarnason, 1991). It rarely produces sporophytes on Iceland, but when it does, the sporophytes are usually abundant (Jóhannsson, 1993). This species can also propagate by detached fragments (2-4 mm) (Bjarnason, 1991; Magnúsdóttir,
2010) and since the plants become fragile when in dry condition it is possible to imagine moss fragments being important for local propagation.

Racomitrium ericoides (17 quadrats) was the third most widespread bryophyte species on Surtsey in 2008 (Fig. 4). It was first discovered on Surtsey relatively late, in 1984, and sampled again in 1990 and 2003. This species is common in Iceland, found all around the country (including Vestmannaeyjar) in various habitats both near and far from the coast. It quite often forms sporophytes (Jóhannsson, 1993) but like its relative $R$. lanuginosum it can also propagate by moss fragments (2-4 mm) (Magnúsdóttir, 2010).

Racomitrium fasciculare (13 quadrats) was in 2008 mostly found around the largest crater Surtungur but also in the southern and southwestern parts of Surtsey (Fig. 4). This species is widespread in Iceland, both near and far from the coast (also in Vestmannaeyjar) and is rather frequently found with sporophytes (Jóhannsson, 1993). It was first discovered on Surtsey in 1975 and was sampled again in 1984, 1990 and 2003. This species is likely a successful colonizer.

Bryum argenteum (11 quadrats; Fig. 4), was mostly found along the palagonite ridge where most of the fumaroles (steam vents) occur (Figs. 1 and 6). This species was first discovered on Surtsey in 1972 with 25 samples out of 63 found in habitats affected by fumaroles. The hydrothermal area has greatly decreased since 1972 (Jakobsson et al., 


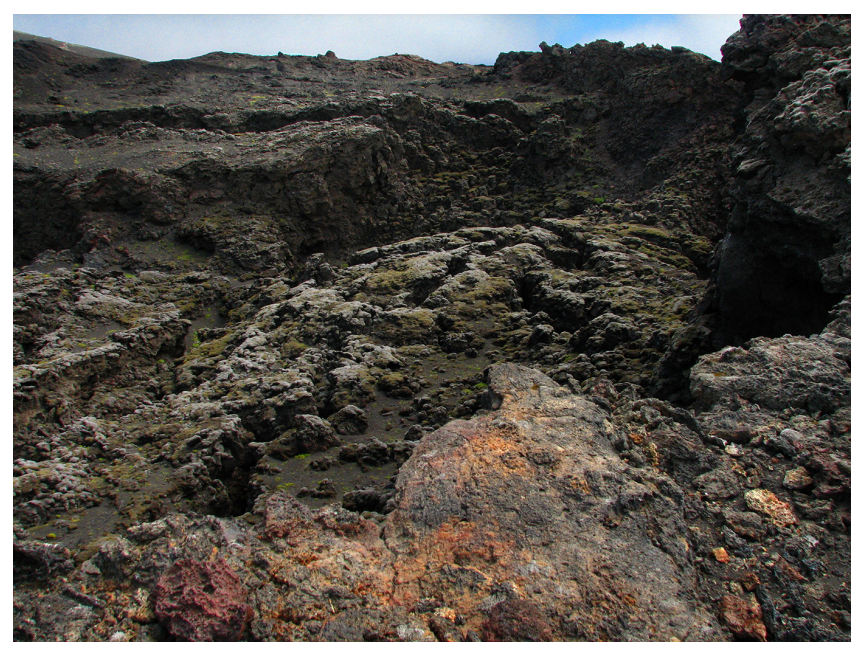

Figure 5. Moss carpet, mostly made up of Racomitrium lanuginosum, in the bottom of Surtungur crater, the largest crater on Surtsey. Photo: GVI, 9 July 2008.

2009) which can partially explain the reduction of the distribution of $B$. argenteum. This species is widespread in Iceland (also present in Vestmannaeyjar) where it rarely carries sporophytes; instead it commonly sheds specialized vegetative propagules (gemmae). The plant itself is only about 2 $15 \mathrm{~mm}$ high and frequently grows in cracks and crevices, in ruderal habitats and on bird cliffs (Jóhannsson, 1995). The species was first encountered on Surtsey in 1967, second only after Funaria hygrometrica (Jóhannsson, 1968) and it has subsequently been found during inventories performed in 10 different years, making it the most frequently encountered bryophyte (Table 1). By 1986 it had formed a crust within the gull colony (P12) (Magnússon et al., 2009), perhaps not surprising given the affinity of the species for disturbed habitats rich with nutrients (Atherton et al., 2010).

Ceratodon purpureus was first discovered on Surtsey in 1968 (Table 1). The distribution in 2008 (10 quadrats, four of which were new for the species; Fig. 4) appears restricted compared to the situation in 1972 (26 of the selected quadrats). This species is common in Iceland (including Vestmannaeyjar) and commonly found with spore capsules. It occurs on naked, rather dry or moist sandy soil (Jóhannsson, 1992a).

Cephaloziella cf. divaricata was the eighth most widespread bryophyte species and by far the most widespread liverwort species found in 2008 (eight quadrats; Fig. 4) despite its small and inconspicuous appearance. It was first discovered in 1972 (Table 1) and then again in 2003 and 2006. Cephaloziella divaricata is widespread in Iceland (also found in Vestmannaeyjar) and often grows near the coast. It rarely produces sporophytes (Jóhannsson, 2000).

Bryoerythrophyllum recurvirostrum (seven quadrats) was the ninth most widespread bryophyte species encountered in 2008 (Fig. 4). It is widespread in Iceland (also found in
Vestmannaeyjar) and is usually found on rocks and sandy soils, but also in lava fields (Jóhannsson, 1992b). It was found on Surtsey in 1971 and 1972, in somewhere between 2 and 10 quadrats (then as Barbula recurvirostra). Note that these quadrats are not restricted to the selected quadrats since information on the exact distribution for this species is lacking for these years except that it was found in P11 with spore capsules (Magnússon and Friðriksson, 1974). It was encountered in 1984 and 1990 (Table 1) and it seems to have permanently colonized Surtsey.

Almost all of the early colonizers are frequently producing sporophytes and they appear to be easily dispersed by windborne spores. They are primary colonizers and several have been shown to carry nitrogen-fixing cyanobacteria (Henriksson et al., 1987).

\subsection{New arrivals}

Since 1972, a total of 23 species have been added to the accumulated list of bryophyte species observed on Surtsey, including additions from the 2008 survey (Table 1). Ulota phyllantha is a relatively late colonizer, first discovered on Surtsey in 2003 (Table 1). In 2008 it was confined to the southern part of Surtsey (11 quadrats; Fig. 4). It is found all around the Icelandic coast (including Vestmannaeyjar). It usually grows on rocks and lava by the sea but can be found further inland as well. Plants are dioecious, but sporophytes are unknown and instead the shoots produce numerous gemmae (5-10 cells length) (Jóhannsson, 1990). Just like Schistidium maritimum it is one of the few bryophytes that can tolerate growing on cliffs temporarily exposed to saline water (Frey et al., 2006).

Altogether 38 bryophyte species were found in 2008, of which four were liverwort species. Eight of the moss species were new to Surtsey and first reported here: Bryum elegans, Ceratodon heterophyllus, Didymodon rigidulus, Eurhynchium praelongum, Schistidium confertum, S. papillosum, Tortula hoppeana and T. muralis (Table 1). All of these were found in single quadrats except $B$. elegans which was found in four quadrats (H11, K18, P13 and Q12). An additional seven observed species had been encountered during the years after 1972. Eight species were neither encountered in 2008 nor 1972 or earlier but were discovered in the years between (Table 1).

The most abundant of the newcomers, Bryum elegans is a rare but relatively widespread species on lava fields in Iceland (also found in Vestmannaeyjar). It has not been found with spore capsules in Iceland (Jóhannsson, 1995). Ceratodon heterophyllus (Fig. 4) has not been reported from Iceland before and the only previous report from Europe until now is from Spitsbergen according to Frey et al. (2006). The specimen collected from Surtsey did not have capsules and in the European checklist for mosses it is noted that all the European specimens assigned to $C$. heterophyllus lack spore capsules, a fact which renders the identification somewhat uncertain (Burley and Pritchard, 1990; Hill et al., 2006). 
Didymodon rigidulus is usually found in the southernmost part of Iceland (including Vestmannaeyjar); it rarely produces spore capsules in Iceland. Eurhynchium praelongum is rare on Iceland, but locally abundant in the southern- and southwesternmost parts of the country (also in Vestmannaeyjar). It grows on soil, for example in moist grasslands a habitat relatively recently established on Surtsey. It was found in quadrat P13, that is, within the gull colony. Just like several of the preceding late newcomers, sporophytes have not been reported from Iceland (Jóhannsson, 1997), which means that they are unlikely to have arrived by winddispersed spores. The most probable alternative hypothesis for their emergence in this phase of the Surtsey colonization is that they have been carried from the mainland of Iceland by the gulls in form of specialized vegetative propagules or as detached leaf or stem fragments.

Only six liverwort species have been identified from Surtsey and all but Cephaloziella cf. divaricata have been rare (Fig. 4). Cephaloziella cf. hampeana, Reboulia hemisphaerica and Scapania sp. (curta or scandica) were found in a single quadrat respectively. Cephaloziella hampeana is even more widespread in Iceland (also found in Vestmannaeyjar) than $C$. divaricata. It grows often in moist grasslands, moist soil in cliffs and geothermal areas but it does not show the same affinity towards the coast as does $C$. divaricata (Jóhannsson, 2000). It is therefore not surprising that this species has not been as successful in colonizing Surtsey.

\subsection{Declining species}

In 1972 Racomitrium canescens was found in 40 of the selected quadrats and was reported as "common" (i.e. presence in 10 or more locations within a quadrat) in 32 of those. The samples were mostly taken from "naked, exposed lava surface without sand" (954 out of 966 samples) (Magnússon and Friðriksson, 1974). Since 1972 no report exists of $R$. canescens at all from Surtsey (Table 1). However, R. ericoides eventually appears in 1984, shortly after a revision of the Racomitrium canescens complex by Frisvoll (1983). Prior to this revision, there was a lot of confusion about how to delimit $R$. canescens against $R$. ericoides and it is therefore likely that samples from Surtsey denoted to $R$. canescens and sampled in 1972 or earlier have indeed been the same species as is now called $R$. ericoides. In fact, there are two specimens in ICEL from Surtsey in 1970 that are identified as $R$. canescens var. ericoides, now a synonym for $R$. ericoides. These identifications were simply published as $R$. canescens. Unlike $R$. ericoides, $R$. canescens has not been found with spore capsules in Iceland (Jóhannsson, 1993, 2003). R. ericoides was found in 17 quadrats in 2008 compared to 40 of the selected quadrats in 1972 (Fig. 4).

Several other species have a drastically reduced distribution: Leptobryum pyriforme was found in 13 of the selected quadrats in 1972 but none in 2008; Schistidium apocarpum coll. was found in 15 of the selected quadrats in 1972 but none in 2008 (although S. papillosum was found in a single quadrat in 2008, and might have been previously assigned to $S$. apocarpum - Grimmia apocarpa in previous reports); Funaria hygrometrica was found in 35 of the selected quadrats in 1972 but in 2008 it was only found in a single quadrat; Philonotis spp. was found in 22 of the selected quadrats in 1972 but only one in 2008; and Pohlia spp. was found in 23 of the selected quadrats in 1972 but only one in 2008. Schistidium strictum was found in two of the selected quadrats in 1971 but in as many as 25 in 1972 (Magnússon and Friðriksson, 1974). In 2008 the species was found in only five quadrats. Most of the declining species are typically growing on base-rich soils or ashes, indicating that they disappear as cations are gradually washed out from the soil by precipitation and erosion.

\subsection{Habitats}

The environment on Surtsey was quite unstable during the years of the eruption (1963-1967) and continued to be so after the eruption had ended. Loose tephra is easily blown to and from, especially on a small island with the open North Atlantic Ocean in all but one direction, where winter storms can be severe (Jakobsson et al., 2007). Such forces as pound on Surtsey evidently make life difficult for primary colonists. In addition, the cooling of the island and the gradual transformation of volcanic material, such as the transition of loose tephra into compact palagonite tuff (Jakobsson et al., 2000), has steadily changed the microhabitats available for colonizing species. For instance, geothermal areas have been cooling down near the surface; south of the western crater surface temperatures dropped from over $350^{\circ} \mathrm{C}$ in 1971 to below $50^{\circ} \mathrm{C}$ in 1998 (Jakobsson et al., 2000).

Bjarnason and Friðriksson (1972) divided the moss growth areas on Surtsey into four classes in 1969, two of which were affected by geothermal activity: (B) Bare lava affected by heat and steam emission; and (D) sandy lava strongly affected by heat and steam emission. In 1969 moss was most common in areas with heat and steam emission (Bjarnason and Friðriksson, 1972). Accordingly, class D had the most extensive moss zonation and the densest moss cover. The most common moss species was Funaria hygrometrica which was found almost everywhere. Bryum argenteum and Leptobryum pyriforme were second most common. Racomitrium canescens was not common and only found on bare lava. Pogonotum urnigerum was found only in one place, in the cinder slopes of Surtungur (Bjarnason and Friðriksson, 1972).

In 1972 Magnússon and Friðriksson (1974) used 12 different habitat categories and made observations of the habitat choices of the bryophyte species on Surtsey and their coverage in these habitats. By far the most common habitat choice was naked lava with 1988 samples out of 4593 coming from that habitat. Two of the habitat types they defined were connected with geothermal activity: one with a thin layer of 
tephra on a lava surface, fully exposed and affected by fumaroles; the other with moist shaded sand, warm from fumaroles. In the first of these habitat types they gathered 191 samples of the 23 most common species (other species excluded) and in the second habitat type they gathered 31 samples, thus altogether 222 samples (5\%) came from areas affected by geothermal activity.

Bryophytes growing on rocks were by far the most prominent group in 2008. Classes B and D from 1969 (Bjarnason and Friðriksson, 1972) were not encountered. Only few steam vents remained on the palagonite tuff cones, which were almost devoid of bryophytes and instead dominated by cyanobacteria or algae growing on the moist, dense ground near the vents (Fig. 6). Bryophytes were predominantly found in small sand-filled crevices in the lava, which provided shelter from wind and drought. One of the most prominent changes between 1972 and 2008 is the establishment of a gull colony in 1984. The gull invasion was followed by a great increase in vascular plants that are assumed to have been dispersed to the island by the gulls (Magnússon et al., 2009). Within the colony, dense vegetation consisting mainly of grasses has developed due to spillover nutrients from the birds (Fig. 1). Some bryophytes have also been favoured by this development, such as Bryum spp. and Schistidium spp., making up dense cushions (Fig. 7). The gull colony and places in the shelter of Surtungur crater are without a doubt the habitats where bryophytes are most abundant. The following species were confined to the quadrats within and nearest to the gull colony (N11N15, O10-O16, P11-P15, Q11-Q15): acrocarpous (cushionforming) mosses like Bryum capillare, Ceratodon heterophyllus, Mnium hornum, Philonotis fontana, Trichostomum brachydontium and Ulota phyllantha; pleurocarpous (matforming) mosses like Brachythecium albicans, Eurhynchium praelongum, Rhytidiadelphus squarrosus and Sanionia uncinata; liverworts such as Cephaloziella hampeana, Reboulia hemisphaerica and Scapania sp. They are thus heterogeneous with respect to growth form - several are halotolerant but few are expressly nitrophilous and most are likely to grow on soil with organic content (Dierssen, 2001), being secondary colonists. It is probable that the accumulation of organic soil and dispersal factors are more important for their colonization than excesses of nitrogen.

Interestingly, in 1990 an analysis of gulls' nest material showed that 49 out 67 nests contained at least some moss, and 31 were solely made up of moss (Petersen, 2009). The gulls used mainly Racomitrium (Magnússon et al., 2009) and probably $R$. lanuginosum, which they gathered for instance in the western crater, Surtungur (B. Magnússon, personal communication, 2013). In 2003 there were about 300 pairs of large gulls in the colony (Petersen, 2009) but mosses are hardly used any longer since grasses, which the gulls prefer, are plentiful (B. Magnússon, personal communication, 2013).

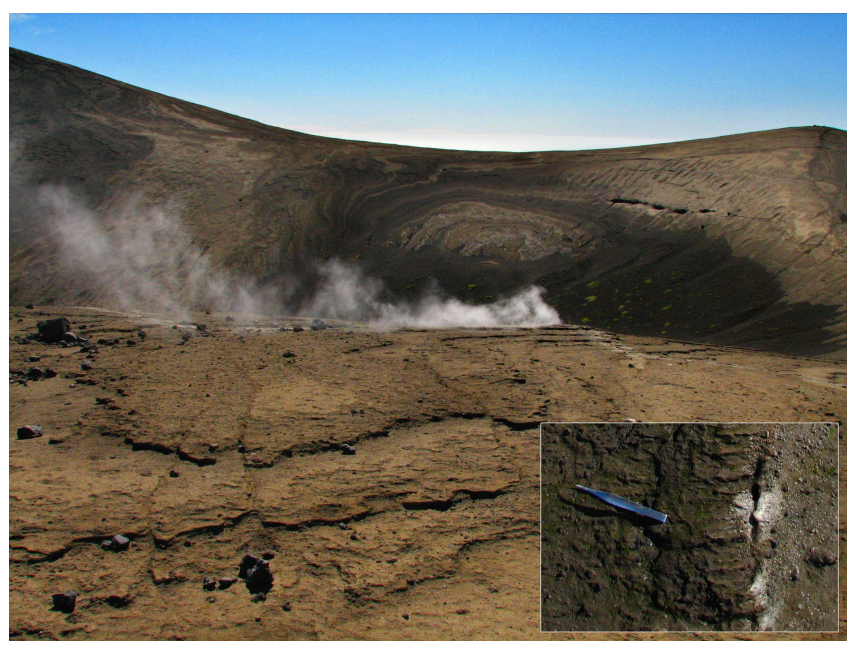

Figure 6. Fumaroles on Surtsey on the western palagonite ridge. The inserted photo shows a close-up of a fumarole, with tweezers for size comparison. Very few bryophytes, mostly Bryum argenteum, were found in this habitat, and then near the fumaroles or in narrow cracks in rock that had cooled off. Photos: GVI, 10 July 2008.

\subsection{Surtsey and other islands of eruptive origin}

There are few places comparable to Surtsey. The tropical Krakatau Islands in Indonesia were subject to a catastrophic volcanic eruption in 1883 which resulted in the islands' biota being destroyed or at least suffering near-destruction (Whittaker et al., 1984; Treub, 1888 in Tagawa et al., 1985). Now the islands are covered with forest. Among these islands is the only other oceanic island apart from Surtsey formed in a volcanic eruption in recent times - Anak Krakatau which emerged in 1929 (Tagawa et al., 1985). Surtsey and Anak Krakatau are remarkably similar in physical appearance but differ greatly in volcanic activity and climate. Surtsey has been dormant since 1967 and is located in the middle of the North Atlantic Ocean at $63^{\circ} \mathrm{N}$; while Anak Krakatau is still active (Badan Geologi, 2014) and is situated between Java and Sumatra at $6^{\circ} \mathrm{S}$.

On Surtsey bryophytes play an important role as primary colonizers and still half a century after the eruption ceased they occupy habitats where few or no vascular plants could otherwise thrive, namely the porous surface of lava rocks. On the other hand, on Krakatau Islands, vascular plants were the primary colonizers after the 1883 eruption. In $1897 \mathrm{sev}$ eral bryophytes were nevertheless found but they seem to have played a minor role in the primary succession (Penzig, 1902 in Whittaker et al., 1984). On Anak Krakatau, Tagawa et al. (1985) studied six lava fields in 1982 that were formed in the years from 1963 to 1979 , and found vascular plants to be pioneer species. In fact they found no plants on two of the oldest fields and do not mention any bryophytes on the others. Bryophytes and lichens are well known as primary 
colonizers on lava fields, for example in Japan, Italy, Hawaii and Iceland (e.g. Tagawa et al., 1985; Miller, 1960).

To find a virgin land more comparable to Surtsey one needs to look to Deception Island $\left(63^{\circ} \mathrm{S}\right)$ in the South Shetland Islands in the Antarctic. The island was subject to volcanic eruptions in 1967, 1969 and 1970. The moss cover development following the eruption was at the time an example of a succession at a considerably faster rate than had been seen elsewhere in the Antarctic (Smith, 1984). The primary colonizers on the volcanic soils of Deception Island were Ceratodon cf. purpureus, Bartramia patens, Sanionia uncinata, Funaria cf. hygrometrica (then new to the Antarctic and restricted to geothermal areas) and Marchantia polymorpha. The colonists vanished as the heated areas cooled down. The exception was a site where heat was diffusing through a vegetated area rather than through a restricted vent. There the bryophytes continued to thrive, forming closed patches of several square metres dominated by Marhcantia berteroana, Campylopus cf. introflexus, Polytrichum juniperinum, Ceratodon cf. purpureus and Leptobryum cf. pyriforme. Bryum argenteum and Tortula excelsa were among the more scattered species. Leptobryum was then new to the Antarctic (Smith, 1984). Interestingly some of the species the island has in common with Surtsey, despite being separated by half the globe. These comprise Ceratodon purpureus, Sanionia uncinata, Funaria hygrometrica, Leptobryum pyriforme, Bryum argenteum and Marchantia polymorpha. The last one has an insignificant role on Surtsey, having only been found twice, while all the others have at least at some point been conspicuous on Surtsey during the primary colonization phase, 1970 or earlier. Only L. pyriforme and M. polymorpha were not encountered on Surtsey in 2008 (Table 1). Funaria hygrometrica, B. argenteum and $C$. purpureus are cosmopolitan and well-known colonizers of sand- or dust-filled cracks and crevices in lava flows (e.g. Miller, 1960). Their ubiquitous presence as primary colonizers on volcanic islands may depend on an ability to grow in substrates with more or less extreme mixtures of basic minerals in combination with a scarcity of nitrogen. Studies from Surtsey have shown that F. hygrometrica, C. purpureus, Bryum sp., Racomitrium canescens, $R$. ericoides and $R$. lanuginosum have cyanobacteria associated with leaves in the upper photosynthetic part of the shoots which fix nitrogen that becomes available to the moss (Henriksson et al., 1987). Protonema of F. hygrometrica has even been found to be associated with a specific cyanobacterium, Anabaena variabilis, on Surtsey to the extent that it may be described as a symbiotic relationship (Schwabe, 1974).

Campylopus cf. introflexus was the very first moss to be found on the Iilewa lava flow, on the tropical Hawaii Island $\left(19^{\circ} \mathrm{N}\right)$. The lava was formed in a large volcanic eruption in 1955 and the moss appeared only about 7 months after the eruption started (Miller, 1960). Campylopus introflexus which was also found on Deception Island is unknown from Surtsey but has been spreading fast in Iceland since it was
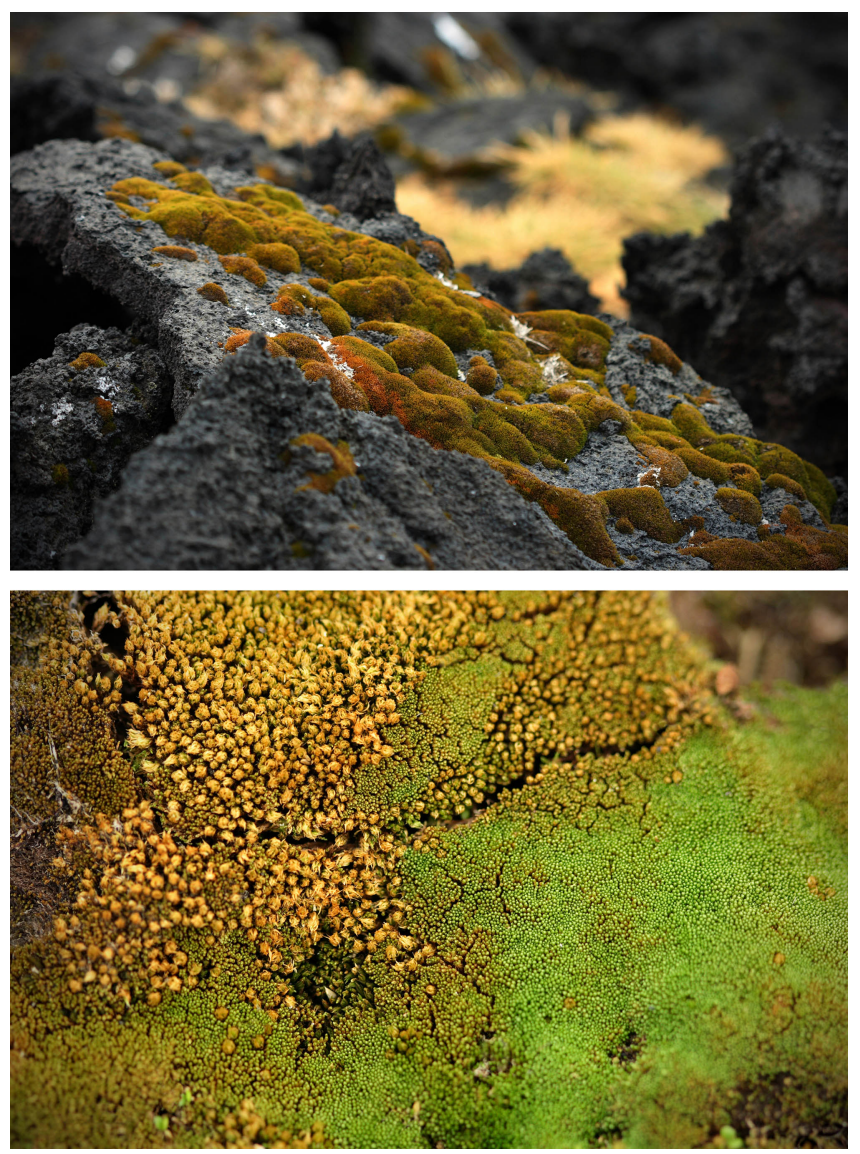

Figure 7. Schistidium spp. (upper image) growing on lava and Bryum spp. (lower image) growing in dense cushions; both within the gull colony. Photos: GVI, 7 July 2008.

first encountered in 1983. Its native range is in the Southern Hemisphere - for instance in Chile, South Africa and New Zealand. In Iceland it is localized in geothermal areas (Klinck, 2010). In Hawaii, Campylopus species are often found on rocks devoid of other plants (Miller, 1960).

\subsection{Comparison with other groups}

The colonization of vascular plants and lichens on Surtsey has been carefully monitored from the beginning. The first three lichen species on Surtsey were recorded in 1970; in 1984 the number was up to 17 and in 200858 lichen species were recorded. The lichen colonization increased considerably right after the establishment of the gull colony on the island in 1984 (Kristinsson and Heiðmarsson, 2009). A similar story can be told for vascular plants. In 1967 there were four species of vascular plants found on Surtsey; in 1984 only 11 species were found but in 200863 vascular plant species were recorded, with colonization benefitting from the development of the gull colony (Magnússon et al., 2009). For comparison, there were two bryophyte species discovered in 1967; in 1984 the number was up to 33 and in 2008 total 
of 43 bryophyte taxa were encountered. It should be kept in mind that both in 1984 and 2008 only partial inventories were performed and due to the scarcity of bryophyte inventories it is difficult to speculate much about the effects of the gull colony of their succession. However, as previously stated, at least some species seem to benefit from the gulls and more species are found within the colony than outside its borders.

\section{Conclusions}

It is apparent that the colonization of bryophytes on Surtsey was relatively fast during the first five years after the eruption ended, and the island does not seem to be significantly isolated, as to hinder the major pioneer species on new volcanic land to arrive at Surtsey, such as Racomitrium spp., Schistidium spp., Funaria hygrometrica and Bryum argenteum. The lack of habitats and substrates is probably a more important factor than dispersal limitation for many colonists, seeing that moss protonema, and nothing else, grew from the ash samples taken in June 1967 (Maguire Jr., 1968). Associations between nitrogen-fixing cyanobacteria and mosses have been essential for the early bryophyte expansion. Bryophyte succession then took a new direction after the formation of the gull colony in 1984 as the bryophytes are affected by gulls in several ways. The gulls transport nutrients to the island, which means that the supply of nitrogen from bryophytes is less critical for the development of the vegetation. The additional nutrients have speeded up the formation of grassland and evidently also locally fuelled the growth of mosses (see Fig. 7). The gulls are likely to contribute to the transportation of bryophyte propagules or gametophyte fragments - several of the recent introductions lack sporophytes on Iceland, suggesting that they are unlikely to be dispersed by windborne spores. Finally, the gulls redistribute moss material when it is used for nest material, although this effect seems to have diminished after the establishment of grasses.

For future research it would be a priority to schedule a more regular and detailed monitoring of species distribution at an interval of no more than 5 years; to study the abiotic and biotic habitat factors of species, including symbionts; and to study the reproductive behaviour of the colonizing bryophyte species, that is, frequency of sporophytes, whether the plants are monoecious or dioecious, and whether propagation is vegetative or sexual. Studying the genetic diversity of selected species would be highly interesting as well since the species' residential time on Surtsey is known. Better knowledge of the bryophyte flora on the neighbouring islands of Vestmannaeyjar Archipelago, which are older but share a volcanic origin with Surtsey, might be most informative regarding successional changes to be expected on Surtsey.
Acknowledgements. The Icelandic Coast Guard transported researchers to and from Surtsey and Toyota Iceland gave financial support to the biological research on Surtsey in 2008. We would like to express our gratitude to the Surtsey Research Society for giving us the opportunity to study bryophytes on Surtsey, and to Borgbór Magnússon for his encouragement and guidance from the very beginning. Skúli Pór Magnússon, first author of the report from the last bryophyte inventory on Surtsey was always ready to help in any way possible. Ellý R. Guðjohnsen extracted information from the databases and herbaria of the Icelandic Institute of Natural History and Anette Theresia Meier provided us with the Surtsey contours and grid for map drawing. Ævar Petersen and Borgbór Magnússon, kindly provided information on the gull colony. They who have collected bryophytes on Surtsey during their visits to Surtsey for other purposes; and made sure they were preserved for future references in the herbaria in Iceland (ICEL and $\mathrm{AMNH}$ ) have provided valuable information; these include Skúli Pór Magnússon, Sigurður H. Magnússon, Kristbjörn Egilsson, Hörður Kristinsson, Hálfdán Björnsson, Eypór Einarsson and Sveinn Jakobsson; only mentioning those who did not have their findings previously published. The editor Borgpór Magnússon, Starri Heiðmarsson and an anonymous referee provided valuable critique on the manuscript. We wish to thank all of the above.

Edited by: B. Magnusson

\section{References}

Atherton, I. D. M., Bosanquet, S. D. S., and Llawley, M. (Eds.): Mosses and Liverworts of Britain and Ireland: a Field Guide, British Bryological Society, 856 pp., 2010.

Badan Geologi: http://www.vsi.esdm.go.id/, last access: 12 June 2014.

Bjarnason, Á. H.: Vegetation on lava fields in the Hekla area, Iceland, Acta Phytogeographica Suecica, 77, 110 pp., 1991.

Bjarnason, Á. H. and Friðriksson, S.: Moss on Surtsey, summer 1969, Surtsey Research Progress Report, 6, 9-10, 1972.

Burley, J. S. and Pritchard, N. M.: Revision of the genus Ceratodon, Bryophyta, 1, 17-76, 1990.

Dierssen, K.: Distribution, Ecological Amplitude and Phytosociological Characterization of European Bryophytes, J. Cramer, Berlin, 289 pp., 2001.

Fonge, B. A., Yinda, G. S., Focho, D. A., Fongod, A. G. N., and Bussmann, R. W.: Vegetation and soil status on an 80 year old lava flow of Mt. Cameroon, West Africa, Lyonia, 8, 19-41, 2005.

Frey, W., Frahm, J.-P., Fischer, E., and Lobin, W.: The Liverworts, Mosses and Ferns of Europe, 512 pp., 2006.

Friðriksson, S.: The Colonization of Vascular Plants on Surtsey in 1968, Surtsey Research Progress Report, 5, 10-14, 1970.

Friðriksson, S. and Johnsen, B.: The colonization of vascular plants on Surtsey in 1967, Surtsey Research Progress Report, 4, 31-39, 1968.

Friðriksson, S., Magnússon, S., and Sveinbjörnsson, B.: Substrate Map of Surtsey 1970, Surtsey Research Progress Report, 6, 6063, 1972.

Friðriksson, S., Sveinbjörnsson, B., and Magnússon, S.: Vegetation on Surtsey - Summer 1970, Surtsey Research Progress Report, 6, 54-59, 1972. 
Frisvoll, A. A.: A taxonomic revision of the Racomitrium canescens group (Bryophyta, Grimmiales), Gunneria, 41, 181 pp., 1983.

Henriksson, L. E. and Henriksson, E.: Concerning the Biological Nitrogen Fixation on Surtsey, Surtsey Research Progress Report, 9, 9-12, 1982.

Henriksson, E., Henriksson, L. E., Norrman, J. O., and Nyman, P. O.: Biological dinitrogen fixation (acetylene reduction) exhibited by blue-green algae (Cyanobacteria) in association with mosses gathered on Surtsey, Iceland, Arctic Alpine Res., 19, 432-436, 1987.

Hill, M. O., Bell, N., Bruggeman-Nannenga, M. A., Brugués, M., Cano, M. J., Enroth, J., Flatberg, K. I., Frahm, J.-P., Gallego, M. T., Garilleti, R., Guerra, J., Hedenäs, L., Holyoak, D. T., Hyvönen, J., Ignatov, M. S., Lara, F., Mazimpaka, V., Muñoz, J., and Söderström, L.: An annotated checklist of the mosses of Europe and Macaronesia, J. Bryol., 28, 198-267, doi:10.1179/174328206X119998, 2006.

Jakobsson, S. P. and Guðmundsson, G.: The marine abrasion of Surtsey, Iceland: areal changes 1967-2002 and future development, Náttúrufræðingurinn, 71, 138-144, 2003 (in Icelandic).

Jakobsson, S. P., Guðmundsson, G., and Moore, J. G.: Geological monitoring of Surtsey, Iceland, 1967-1998, Surtsey Res., 11, 99 108,2000

Jakobsson, S. P., Magnússon, B., Ólafsson, E., Porvarðardóttir, G., Gunnarsson, K., Baldursson, S., and Petersen, Æ.: Nomination of Surtsey for the UNESCO World Heritage List, edited by: Baldursson, S. and Ingadóttir, Á., Icelandic Institute of Natural History, Reykjavík, 123 pp., 2007.

Jakobsson, S. P., Thors, K., Vésteinsson, Á. T. H., and Ásbjörnsdóttir, L.: Some aspects of the seafloor morphology at Surtsey volcano: The new multibeam bathymetric survey of 2007, Surtsey Res., 12, 9-20, 2009.

Jóhannsson, B.: Bryological observation on Surtsey, Surtsey Research Progress Report, 4, 6, 1968.

Jóhannsson, B.: Icelandic bryophytes - Archidiaceae, Splachnaceae and Orthotrichaceae, Fjölrit Náttúrufræðistofnunar, 15, 80, 1990 (in Icelandic).

Jóhannsson, B.: Icelandic bryophytes - Pottiaceae, Fjölrit Náttúrufræðistofnunar, 21, 120, 1992a (in Icelandic).

Jóhannsson, B.: Icelandic bryophytes - Ditrichaceae, Bryoxiphiaceae, Fissidentaceae and Seligeriacea, Fjölrit Náttúrufræðistofnunar, 20, 76, 1992b (in Icelandic).

Jóhannsson, B.: Icelandic bryophytes - Grimmiaceae, Fjölrit Náttúrufræðistofnunar, 24, 116, 1993 (in Icelandic).

Jóhannsson, B.: Icelandic bryophytes - Bryaceae, Fjölrit Náttúrufræðistofnunar, 27, 162, 1995 (in Icelandic).

Jóhannsson, B.: Icelandic bryophytes - Brachytheciaceae, Fjölrit Náttúrufræðistofnunar, 33, 83, 1997 (in Icelandic).
Jóhannsson, B.: Icelandic bryophytes - Lophoziaceae, Gymnomitriaceae and Cephaloziellaceae, Fjölrit Náttúrufræðistofnunar, 41, 151, 2000 (in Icelandic).

Jóhannsson, B.: Icelandic bryophytes - Lists and additions, Fjölrit Náttúrufræðistofnunar, 44, 135, 2003 (in Icelandic).

Klinck, J.: NOBANIS - Invasive Alien Species Fact Sheet - Campylopus introflexus, Online Database of the European Network on Invasive Alien Species - NOBANIS, available at: www.nobanis. org (last access: 27 December 2013), 2010.

Kristinsson, H. and Heiðmarsson, S.: Colonization of lichens on Surtsey 1970-2006, Surtsey Res., 12, 81-104, 2009.

Magnúsdóttir, M.: Leiðir til að fjölga mosum, einkum hraungambra (Racomitrium lanuginosum), The Agricultural University of Iceland, available at: http://hdl.handle.net/1946/7208, 2010.

Magnússon, B., Magnússon, S. H., and Friðriksson, S.: Developments in plant colonization and succession on Surtsey during 1999-2008, Surtsey Res., 12, 57-76, 2009.

Magnússon, S. and Friðriksson, S.: Moss vegetation on Surtsey in 1971 and 1972, Surtsey Research Progress Report, 7, 45-57, 1974.

Maguire Jr., B.: The Early Development of Freshwater Biota on Surtsey, Surtsey Research Progress Report, 4, 83-88, 1968.

Miller, H. A. A.: Remarks on the Succession of Bryophytes on Hawaiian Lava Flows, Pac. Sci., 14, 246-247, 1960.

Penzig, O.: Die Fortschritte der Flora des Krakatau, Ann. Jard. Bot. Buitenz, 18, 92-113, 1902.

Petersen, Æ.: Formation of a bird community on a new island, Surtsey, Iceland, Surtsey Res., 12, 133-148, 2009.

Schwabe, G. H.: Nitrogen fixing blue-green algae as pioneer plants on Surtsey 1968-1973, Surtsey Research Progress Report, 7, 22 25, 1974.

Smith, R. I. L.: Colonization and recovery by cryptogams following recent volcanic activity on Deception Island, South Shetland Islands, Brit. Antarct. Surv. B., 62, 25-51, 1984.

Tagawa, H., Suzuki, E., Partomihardjo, T., and Suriadarma, A.: Vegetation and succession on the Krakatau Islands, Indonesia, Vegetatio, 60, 131-145, 1985.

Treub, M.: Notice sur la nouvelle flore de Krakatau, Ann. Jard. Bot. Buitenz, 7, 215-223, 1888.

Whittaker, R. J., Richards, K., Wiriadinata, H., and Flenley, J. R.: Krakatau 1883 to 1983: a biogeographical assessment, Prog. Phys. Geog., 8, 61-81, doi:10.1177/030913338400800103, 1984.

Pórarinsson, S.: The Surtsey Eruption: Course of Events and the Development of the New Island, Surtsey Research Progress Report, 1, 51-55, 1965. 